\title{
Device Identification Using Active Noise Interrogation and RF-DNA "Fingerprinting" for Non-Destructive Amplifier Acceptance Testing
}

\author{
Mathew Lukacs, Peter Collins and Michael Temple \\ Department of Electrical and Computer Engineering \\ The Air Force Institute of Technology \\ Wright-Patterson AFB, OH 45433 \\ Email: mathew.lukacs@afit.edu
}

\begin{abstract}
The cost of quality is critical to all industrial processes including microwave device production, which is often labor intensive and subject to production defects. Early defect detection can improve quality and reduce cost. A novel approach to defect detection has been demonstrated using a random noise radar (RNR), coupled with Radio Frequency Distinctive Native Attributes (RF-DNA) fingerprinting processing algorithms to non-destructively interrogate microwave devices. The RNR is uniquely suitable since it uses an Ultra Wideband (UWB) noise waveform as an active interrogation method that will not cause damage to sensitive microwave components and multiple RNRs can operate simultaneously in close proximity, allowing for significant parallelization of defect detection systems. Previous experimentation has demonstrated the ability to discern antenna loads and fault conditions, and identify faulty elements in a phased array antenna. This paper extends this method into identifying faulty conditions of devices behind a receive antenna such as typical microwave amplifiers. This method can be used during amplifier production to quickly identify and isolate faulty device production.
\end{abstract}

Index Terms-RF-DNA, Noise, Radar, Amplifier, Classification

\section{INTRODUCTION}

Radio Frequency Distinct Native Attribute (RF-DNA) is a method to extract features received from a Radio Frequency (RF) signal [1]. Every device emits signals that have unique characteristics that can be used to distinguish that device from other, similar devices [2]. This is referred to as "fingerprinting" because the concept resembles human fingerprinting, in which a person's fingerprints distinguish them from another person [1]. RF-DNA fingerprinting has been in development at Air Force Institute of Technology (AFIT) since 2006 successfully demonstrating feature extraction from RF emissions received from various devices including IEEE 802.11 WiFi, IEEE 802.15 BlueTooth, IEEE 802.16 WiMAX, Global Systems for Mobile Communications (GSM) cell phones, Radio Frequency Identification (RFID) emitters [3] and now various RF emitting devices [4], [5], [6], [7]. This last class of devices (those that do not normally emit RF radiation, such as properly shielded RF amplifiers, mixers, oscillators and filters) can be classified using an "active" interrogation method, such as a radar transmission. The RF energy of the radar will be reflected by the device in a manner that can be utilized by RF-DNA fingerprinting analysis. This method can wirelessly extract features from the devices that can be used for quality control such as fault detection, and production lot identification.

\section{BACKGROUND INFORMATION}

\section{A. Noise Radar Overview}

A noise radar transmits a randomly generated noise waveform as opposed to a time harmonic signal a traditional radar transmits to actively interrogate targets. Noise radars are typically characterized by their stochastic and spectral properties such that the interrogation signal is White Gaussian Noise (WGN) [8]. This radar interrogation signal provides several major benefits to include high immunity to noise and spectral compliance (enabling it to function in both high Radio Frequency Interference (RFI) environments and in close proximity to other Random Noise Radar (RNR)s). Many different applications of noise radar technology relevant to the wireless community have been presented over the years ranging from through-the-wall sensing and buried object detection to indoor navigation [9], [10], [11], [12], [13], [14], [15]. It has also demonstrated the ability to detect production anomalies during the construction of microwave systems [5], [6], [4]. The high immunity to noise and spectral compliance allow the RNR to function in the high RFI environment of a typical production floor and noise immunity allows for significant parallelization of production processing. The AFIT Noise Network (NoNet) RNR used in this research is based on an 8-bit, $1.5 \mathrm{GS} / \mathrm{s}$ Analog to Digital Converter (ADC) and all-digital receiver to implement a base-band correlation detection scheme for each radar node.

\section{B. RF-DNA Fingerprinting}

Statistical RF-DNA fingerprints consist of features that are generated by the statistical properties of fixed regions within a received signal [1]. The RF-DNA fingerprint process extracts features using three instantaneous responses: the Instantaneous Amplitude (IA) response, $a(n)$, the Instantaneous Phase (IP) response, $\phi(n)$, and the Instantaneous Frequency (IF) response, $f(n)$. The standard deviation $(\sigma)$, variance $\left(\sigma^{2}\right)$, skewness $(\gamma)$ and kurtosis $(\kappa)$ are generated for each response. 
These statistics are calculated using the following equations:

$$
\begin{gathered}
\sigma^{2}=\frac{1}{N_{x}} \sum_{n=1}^{N_{x}}\left(\bar{x}_{c}(n)-\mu\right)^{2}, \\
\gamma=\frac{1}{N_{x}} \sigma^{3} \sum_{n=1}^{N_{x}}\left(\bar{x}_{c}(n)-\mu\right)^{3}, \\
\kappa=\frac{1}{N_{x}} \sigma^{4} \sum_{n=1}^{N_{x}}\left(\bar{x}_{c}(n)-\mu\right)^{4}
\end{gathered}
$$

where $\bar{x}_{c}(n)$ is the normalized data sequence with $N_{x}$ samples, and each of the above statistics is calculated over an equal, contiguous, sub-region of $\bar{x}_{c}$. These sub-regions are determined empirically and are application specific. For the amplifier classification experiment in section IV, 16 equal subregions were used. In each of these sub-regions, the three statistics calculated in (1), (2) and (3) are concatenated to form a "distinct native attribute marker" to the sub-regional RF-DNA vector, $i$, according to [1]:

$$
F_{R_{i}}=\left[\sigma_{R_{i}} \sigma_{R_{i}}^{2} \gamma_{R_{i}} \kappa_{R_{i}}\right]_{1 x 4} .
$$

After which, each distinctive native attribute marker vector is concatenated to form a "composite characteristic vector" according to [1]:

$$
F^{C}=\left[F_{R_{1}} \vdots F_{R_{2}} \vdots \cdots F_{R_{N_{R}+1}}\right]_{1 x 4\left(N_{R}+1\right)},
$$

in which $C$ denotes the analyzed response (IA, IP or IF), and $N_{R}$ is the total number of sub-regions. Each of the composite characteristic vectors are finally concatenated into one statistical fingerprint (of 60 elements for 5 sub-regions) according to [1]:

$$
F=\left[F^{a} \vdots F^{\phi} \vdots F^{f}\right]
$$

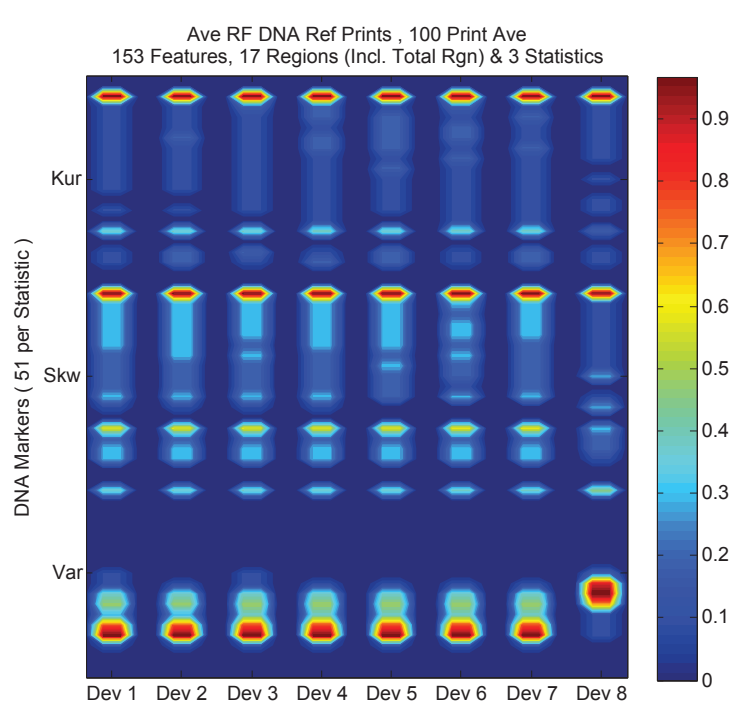

Fig. 1. Visualization of RF-DNA for 8 Devices

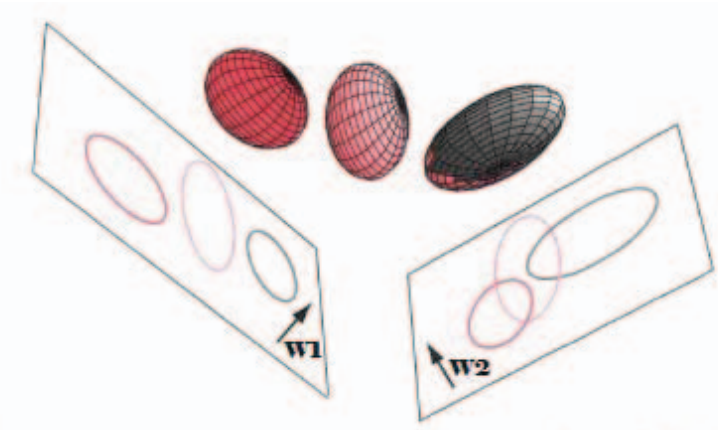

Fig. 2. MDA/ML classification of fingerprint in a 3-class problem [17]

Figure 1 visually depicts some derived RF-DNA fingerprints. This is for visualization purposes only, and is not used for classification, but clearly shows how the extracted features are analogous to human DNA markers. The figure shows the prints for 8 different devices (or classes). The three statistics used, $\kappa, \gamma$, and $\sigma^{2}$ can be seen in the horizontal bands "Kur", "Skw" and "Var" respectively, with the values for each quantization marker stacked vertically. The colors represent the average value for each statistic across 100 prints, scaled to span (0 to 1) according to the legend on the figure right [16].

\section{Multiple Discriminant Analysis / Maximum Likelihood}

Multiple Discriminant Analysis (MDA) is not, by itself, a classifier [17]. It is an algorithm to achieve dimensionality reduction of a multi-dimensional dataset such that a more traditional classifier can be performed (i.e., Maximum Likelihood (ML)). MDA aims to project from a $d$-dimensional space to a $(c-1)$ dimensional space, where $c$ is the number of distinct classes (it is assumed the $d \geq c$, or some space of dimension greater than $c$ ). Figure 2 is a example of MDA/ML applied to a 3-dimensional dataset. In the figure, the MDL algorithm is used to project the 3-dimensional space into two, 2-dimensional sub-spaces. The 2-D sub-spaces are defined by their norm vectors, $W_{1}$ and $W_{2}$ respectively. The algorithm attempts to find the optimal sub-space for classification, by finding one with the greatest separation of the projected distributions of the classes. For instance, discrimination along the $W_{1}$ sub-space would be considerably easier than across the $W_{2}$ sub-space that still has overlapping projections. Obviously, for higher class problems, it becomes difficult to visualize in a 3-dimensional space. For the experiments conducted below, half of the fingerprints are used for model training (i.e., creating of the optimal sub-space) and half are reserved for model validation.

Additional information on RF-DNA and Multiple Discriminant Analysis / Maximum Likelihood (MDA/ML) can be found in [1], [2], [16], [17], [5], [6], [4].

\section{METHODOLOGY}

A typical superheterodyne receiver contains an antenna, an RF amplifier(s), a mixer, local oscillator and some filtering, with a low-noise RF amplifier usually being the first 


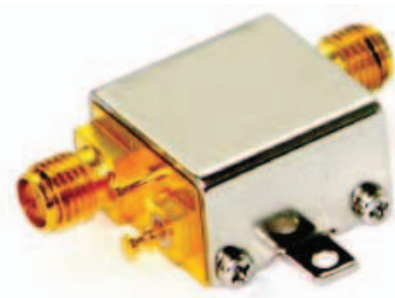

Fig. 3. Typical ZX60 series RF Amplifier used for most of the experimentation

component in the receive chain after the antenna. A typical transmitter configuration will also have an amplifier connected to an antenna so in both configurations, an amplifier is often the first device immediately preceding or following the antenna. This paper focuses on device discrimination (to the production lot level) and classifying the various states (power on vs power off) or termination conditions (Open vs Load) of a representative RF-amplifier. For the tests conducted, three types of amplifiers were chosen: a Mini-Circuits model ZX60-14012L+ amplifier, a mini-circuits ZX60-8008E-S+, and an HD Communications Corp HD28429 Rev G . The first amplifier has approximately $9-12 \mathrm{~dB}$ of Gain and an UWB operational bandwidth of $30 \mathrm{KHz}-14 \mathrm{GHz}$, the second has 9$11 \mathrm{~dB}$ Gain and an operational bandwidth of $20 \mathrm{MHz}$ to $8 \mathrm{GHz}$ and the high power amp with a P1dB of $25 \mathrm{~W}$ and bandwidth of 400 to $1000 \mathrm{Mhz}$. Two different interrogation setups were analyzed. The first interrogated an amplifier with the receive antenna connected to the RF Input, and a termination on the output. This is considered to be the "best case" scenario for this methodology since the amplifier gain is realized before reflection off the termination. The second setup was the reverse configuration with the antenna on the RF output which more closely resembles a typical transmitter architecture. For this configuration, the interrogation signal has to propagate backward through the amplifier before reflection off the termination. The ZX60-14012L+ amps were interrogated using both configurations. The ZX60-14012L+ and HD28429 Rev $\mathrm{G}$ amplifiers will be tested only in the second configuration because it is viewed as the more "real world" configuration. An image of a typical ZX60 RF amplifier is shown in Fig. 3.

\section{A. Experimental Setup}

For the first setup, representing a receiver architecture, the Amplifier Under Test (AmUT) was connected to a typical $2-18 \mathrm{GHz}$ horn antenna and interrogated with a noise signal centered at $7 \mathrm{GHz}$ with $350 \mathrm{MHz}$ bandwidth. This interrogation signal was chosen because the amplifier peak gain occurs at $6-7 \mathrm{GHz}$. The second setup, representing a transmitter architecture, used multiple interrogation frequencies (1.47, $2.45,3.51,4.51$ and $5.52 \mathrm{GHz}$ ) with a noise bandwidth of approximately $1 \mathrm{GHz}$. The tests were conducted in a small anechoic chamber with no additional RFI present, although the noise interrogation signal has previously demonstrated high immunity to RFI down to -20dB 20dB Signal-to-Noise Ratio

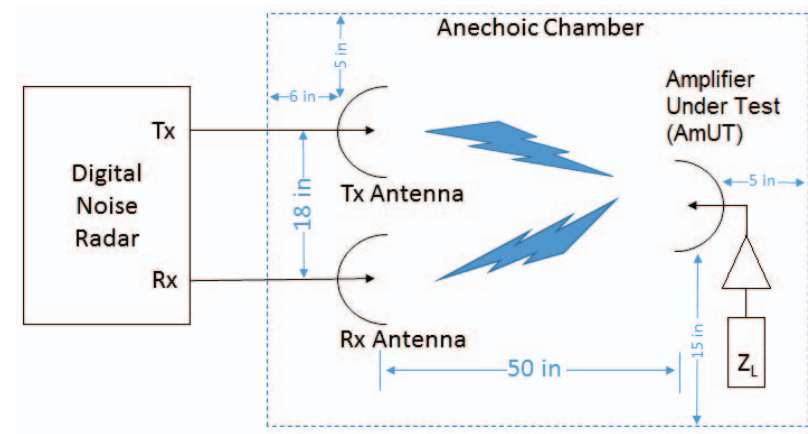

Fig. 4. Experimental setup for the amplifier interrogations. Note that the direction of the amplifier is flipped with Setup 2 shown and Setup 1 flipped.

(SNR) [4], [5], [6], [7]. The AmUT was connected to three different terminations (an "Open", "Short" and $50 \Omega$ Load) and interrogated in both the "on" and "off" state. Five ZX60$1012 \mathrm{~L}+$ amplifiers were tested (including one that was known to be faulty) as well as $14 \mathrm{ZX60-8008E-S+} \mathrm{amplifiers} \mathrm{(pulled}$ from 3 different production lots) and two HD28429 amplifiers. The five ZX60-1012L+ and two HD28429 amplifiers were pulled from research stock and have an unknown amount of operational usage, but the 14 ZX60-8008E-S+ amplifiers were procured new specifically for this test in an attempt to classify production lot variation. A schematic of the experimental setup is shown in Fig. 4.

\section{B. Data Analysis}

Using the AFIT RNR, interrogations of each amplifier in each power state with each termination at each interrogation frequency were conducted. Each of these configurations is regarded as a separate "device" for discrimination purposes (not to be confused with individual amplifiers). The RNR was run for 10 seconds per interrogation, which provides sufficient data to collect approximately 600 "bursts" used to generate 600 fingerprints per device. MDA/ML classification was conducted using half the fingerprints for model training and half for testing with $\mathrm{K}=5$ cross-fold validation. Additionally, 10 model classification runs were averaged for the data presented. Classification is a one-to-many "best match" assessment [18] wherein the fingerprints from an unknown device are compared against a set of fingerprints from several known devices, and the unknown will be identified as one of the known devices based on a chosen estimator. Therefore, classification will always give a result, even though it might not be a good result (e.g., an "apple" might classify as an "orange" because the only options presented were oranges, pears and bananas). A class is one or more devices that can be used for model development to determine classification. Initially (primarily for the ZX60-14012L+ amplifiers), each "device" is regarded as its own class. Specific classification models were developed for each desired classification scenario. For example, to classify the termination condition on a specific amplifier, a classification model is developed using fingerprints for each termination condition on that amp (each 
TABLE 1

\begin{tabular}{|c|c|c|}
\hline Amplifier & \multicolumn{2}{|c|}{ Classified Correctly } \\
\hline Amplifier 1 Load Termination & $72.7 \%$ & $66.3 \%$ \\
\hline Amplifier 1 Open Termination & $72.2 \%$ & $60.1 \%$ \\
\hline Amplifier 1 Short Termination & $86.7 \%$ & \\
\hline Amplifier 2 Load Termination & $91.7 \%$ & $67.2 \%$ \\
\hline Amplifier 2 Open Termination & $86.7 \%$ & $62.3 \%$ \\
\hline Amplifier 2 Short Termination & $78.7 \%$ & \\
\hline Amplifier 3 Load Termination & $72.2 \%$ & $63.2 \%$ \\
\hline Amplifier 3 Open Termination & $78.4 \%$ & $55.7 \%$ \\
\hline Amplifier 3 Short Termination & $94 \%$ & \\
\hline Amplifier 5 Load Termination & $53.1 \%$ & $53.2 \%$ \\
\hline Amplifier 5 Open Termination & $55.6 \%$ & $52.7 \%$ \\
\hline Amplifier 5 Short Termination & $56.2 \%$ & \\
\hline
\end{tabular}

termination being a separate "class"). An alternative method, which is used more extensively with the other amplifier experiments, is to develop a reference class using prints from multiple devices, and then develop a model that classifies other devices against the reference class. For example, to identify production lot discriminability, all of the fingerprints for all the devices in a specific production lot (defined as a "class") are pooled together and a classification model is developed versus another production lot(s) ("classes"). The fingerprints are pooled together by combining all the "bursts" from each device into one large data array (i.e., instead of 600 "bursts" from 3 devices, there will be 1800 "bursts" from 1 device). Interleaving of the fingerprints was performed to ensure model training and validation used prints from all pooled devices.

\section{Amplifier Test Results}

The first interrogation, using setup 1(the receiver configuration) attempted to specifically identify each of the four amplifiers with power on under each of the termination conditions. The results were $72.4 \%$ correct classification for the Load termination, $56.6 \%$ for the Open and $71.9 \%$ for the Short. A random guess would be $25 \%$ (4 amplifiers for a 4class scenario) therefore we can achieve a measure of "serial number level" discrimination of devices behind the antenna. The second interrogation attempted to identify power state ("on" vs. "'off") for each amplifier under the three termination conditions. The results are shown in Table 1 (under Setup 1). The results for amplifiers 1-3 clearly indicate the ability to discern "on" vs "off" for each device, but the amplifier 5 results were similar to a random guess $(50 \%$ for 2 -class scenario) indicating this amplifier had faulty performance. The faulty performance was validated using a Vector Network Analyzer and comparing the S21 parameters in both the "off" and "on" state. Note, for these experiments, amplifier 4 was not used (but will be used later).

Since amplifier 5 tested as faulty, the final interrogation, to discriminate between termination conditions for each amplifier in both power states was only conducted on amplifiers 1-3. Only the Open and Load terminations were chosen because the Short termination tends to confuse with the Open decreasing overall classification and the objective is identify a fault
TABLE 2

Summary of Amplifier Termination State Interrogations

\begin{tabular}{|c|c|c|}
\hline Amplifier & \multicolumn{2}{|c|}{ Classified Correctly } \\
& Setup 1 & Setup 2 \\
\hline Amplifier 1 “Off" & $99.9 \%$ & $90.8 \%$ \\
Amplifier 1 "On" & $99 \%$ & $93.1 \%$ \\
Amplifier 2 "Off" & $99.8 \%$ & $88.1 \%$ \\
Amplifier 2 "On" & $99.5 \%$ & $89.1 \%$ \\
Amplifier 3 "Off" & $97.2 \%$ & $90.8 \%$ \\
Amplifier 3 "On" & $96.2 \%$ & $91.7 \%$ \\
\hline
\end{tabular}

(simulated with an Open) versus normal (simulated with a load). The results are shown in Table 2. For this 2-class scenario, a random guess would be $50 \%$ so the results indicate that classification not only exceeds a random guess, but the system is near perfect at identifying the amplifier termination (when presented with just two choices for classification).

Next, the experiment was repeated using the second setup configuration. Only the Load and Open terminations were used for sake of expediency given the prior analysis. The results are shown in the Setup 2 column of Tables 1 and 2. Both the power state discrimination (Table 1) and the termination state discrimination (Table 2) have decreased, with the power state discrimination having a much larger increase than the termination state. This setup appears to be very good at identifying a faulty termination, but not necessarily identifying a specific device. This is consistent with the fact we are running the amplifier "backward" and therefore the device specific signal path coloration is not as apparent.

Using the same data from Setup 2, an alternative analysis can be conducted wherein the fingerprints from the first 3 known functional devices can be pooled into a single reference class. Then two unknown devices (amplifier 4 and 5 - with 5 being known faulty) are classified against the reference class. For MDA/ML to function properly, each class must have the same number of fingerprints. So only $1 / 3$ of the prints from each of the 3 devices in the reference class were included. To validate repeatability, the reference class is repeated with all combinations of the first 4 amplifiers (with 1 held as a known "good" and amplifier 5 as a known "fault"). The results are summarized in Table 3. The percents indicated are "percent correct" numbers in a 2-class model (i.e, Amp 4, which was known "good" classified $62.3 \%$ of the time correctly versus the reference class of Amps 1-3 which classified correctly at $68.9 \%$ ). Since the reference class is comprised of known functional amplifiers, an ideal scenario would be near 50$50 \%$ classification versus the functional device (meaning the functional device is indistinguishable from the reference class) and near $100 \%$ for the known faulty device. For all 4 scenarios, the faulty device was easily determined in the near $85 \%$ classified correctly range.

The next experiment used the High Power amplifiers in the Setup 2 configuration with the intent of proving "on" vs "off" operation. This experiment was conducted for the purpose of demonstrating method functionality on amplifiers outside the mini-circuits ZX60 production line. Two amplifiers 
TABLE 3

Identifying Faulty vs Functional Amp with Reference Class (\% correct)

\begin{tabular}{|c|c|c|}
\hline Reference Class & Functional Device & Faulty Device \\
\hline Amps 1-3 & Amp 4 & Amp 5 \\
$68.9 \%$ & $62.3 \%$ & $84 \%$ \\
$80 \%$ & & Amp 5 \\
\hline Amps 2-4 & Amp 1 & \\
$74.7 \%$ & $76.5 \%$ & $85.4 \%$ \\
$79.9 \%$ & & Amp 5 \\
\hline Amps1,3-4 & Amp 2 & $84.8 \%$ \\
$54 \%$ & $59.8 \% \%$ & Amp 5 \\
$80.2 \%$ & & $85.2 \%$ \\
\hline Amps 1-2,4 & Amp 3 & \\
$55.8 \%$ & $65.5 \%$ & \\
$81.2 \%$ & & \\
\hline
\end{tabular}

were interrogated using a noise signal from 300-750 $\mathrm{MHz}$ bandwidth. The test had one significant difference in that UWB Log Periodic Antenna (LPA) antennas were used for both the RNR transmit and receive antennas, as well as the AmUT antenna. This was necessary because the operational bandwidth of the amplifiers were below the $2-18 \mathrm{GHz}$ frequency range of the horn antennas used previously. For this test, the fingerprints from both amps were pooled to create separate "on" and "off" reference classes. The resulting MDA/ML classification model achieved $73.4 \%$ correctly classified for the "on" state and $72.9 \%$ for the "off" state which is a little higher performance than the results shown for the similar experiment in Table 1. The higher performance is probably due to the increased reflected signal power as it propagates back through the amplifier.

The final experiment attempted to demonstrate production lot discrimination using a set of 14 brand new amplifiers. Of the 14 devices, 6 were from production lot 1, 6 from production lot 2 and 2 from production lot 3 (unfortunately the supplier could not provide 6 from lot 3). Interrogations are performed using Setup 2 as before. 14 different amplifiers with 2 terminations (open and load), and 2 power states ("on" and "off") result in 56 devices that can be pooled into classes as appropriate. Since MDA/ML prefers all classes to have the same number of fingerprints, the initial analysis will look only at the two lots of 6 amplifiers, with each lot being a single class. The following scenarios were chosen: "on" with a Load termination, "on" with an Open termination, "off" with a load termination, "off" with an open termination, "on" versus "off" and "open" versus "load" terminations. A 2class MDA/ML model was created for each scenario with the percent classified correctly results shown in Table 4 . The results indicate a moderate ability to discriminate production lots in the "On" with Load termination scenario and the "off" with Open termination scenario ( $50 \%$ is a random guess). However, this experiment shows a slightly higher ability to discern the power state of the amplifiers in each lot and just as in previous experiments, it shows an incredibly high ability to determine the termination.

The final data analysis conducted was to include the fingerprints from lot 3 . Rather than reduce the number of prints
TABLE 4

Amplifier production lot discrimination (2-class MDAML \% correct)

\begin{tabular}{|c|c|c|}
\hline Scenario & Lot 1 & Lot 2 \\
\hline "On" w/Load & $72.3 \%$ & $64.4 \%$ \\
"On" w/Open & $60.5 \%$ & $58.1 \%$ \\
"Off' w/Load & $54.4 \%$ & $53.4 \%$ \\
"Off" w/Open & $68.9 \%$ & $68.5 \%$ \\
"On" vs "Off" & $78 \%$ & $77.6 \%$ \\
Open vs Load & $99.98 \%$ & $100 \%$ \\
\hline
\end{tabular}

TABLE 5

Amplifier production lot discrimination (3-class MDAML \% correct)

\begin{tabular}{|c|c|c|c|}
\hline Scenario & Lot 1 & Lot 2 & lot 3 \\
\hline "On" w/Load (Devices 1-2) & $46 \%$ & $57.5 \%$ & $44.7 \%$ \\
"On" w/Open (Devices 1-2) & $49 \%$ & $59 \%$ & $55.7 \%$ \\
"Off' w/Load (Devices 1-2) & $37.3 \%$ & $36 \%$ & $38.9 \%$ \\
"Off" w/Open (Devices 1-2) & $39.2 \%$ & $62.8 \%$ & $46.7 \%$ \\
"On" w/Load (Devices 3-4) & $43.7 \%$ & $67 \%$ & $48.3 \%$ \\
"On" w/Open (Devices 3-4) & $73.4 \%$ & $58.5 \%$ & $61.9 \%$ \\
"Off' w/Load (Devices 3-4) & $39.9 \%$ & $38.4 \%$ & $35.2 \%$ \\
"Off" w/Open (Devices 3-4) & $76.7 \%$ & $47.9 \%$ & $54.3 \%$ \\
"On" w/Load (Devices 5-6) & $34.9 \%$ & $38 \%$ & $33.2 \%$ \\
"On" w/Open (Devices 5-6) & $72.4 \%$ & $60.3 \%$ & $56 \%$ \\
"Off" w/Load (Devices 5-6) & $38.9 \%$ & $41.2 \%$ & $35.58 \%$ \\
"Off" w/Open (Devices 5-6) & $100 \%$ & $54.8 \%$ & $54.4 \%$ \\
\hline
\end{tabular}

from each of the 6 devices in lots 1 and 2, another method was employed where lot 3 is compared to 2 devices from each of lot 1 and 2 (devices 1-2, 3-4 and finally 5-6). The results are summarized in Table 5. The results show a high degree of variability in the resulting data. However, given that a random guess would be $33 \%$ for a 3-class model, most of the scenarios demonstrated a lever of discrimination performance. The "off" state with a load termination had very poor performance approaching a random guess, but the open termination (simulated fault) states had generally better performance. Therefore, based on these results, we believe there is potential for this method to be able to classify devices from different production lots given some specifics on the termination and amplifier state.

\section{CONCLUSION}

The amplifier classification experiments conducted in this work indicate the ability to classify devices within a receiver chain using an active UWB noise signal for interrogation coupled with RF-DNA classifier tools. The ability to identify power state and termination state for multiple amplifiers was demonstrated as well as a capability to determine device production lot. Further analysis will extend this research into additional amplifiers and full receivers (such as hand held radios) as well as additional classification methods.

\section{REFERENCES}

[1] R. Klein, M. Temple, and M. Mendenhall, "Application of waveletbased RF fingerprinting to enhance wireless network security," Journal of Communications and Networks, vol. 11, no. 6, pp. 544-54, Dec 2009.

[2] A. Zeqolari, "Ultra-Wideband Radio Frequency Fingerprinting," M.S. thesis, Air Force Institute of Technology, Dayton, OH, Mar. 2014.

[3] D.R. Reising, "Exploitation of RF-DNA for Device Classification and Verification Using GRLVQI Processing," M.S. thesis, Air Force Institute of Technology, Dayton, OH, Dec 2012. 
[4] M.W. Luakcs, P.J. Luakcs*, and M.A. Luakcs**, "RF-DNA fingerprinting for antenna classification," Antennas and Wireless Propagation Letters, IEEE, vol. 14, pp. 1455-1458, 2015.

[5] M.W. Lukacs, P.J. Collins, and M.A. Temple, “"RF-DNA” fingerprinting for non-destructive antenna acceptance testing," in Proceedings of the Antenna Measurement Techniques Association 36th Annual Symposium, Oct 2014.

[6] M.W. Lukacs, P.J. Luakcs*, and M.A. Luakcs**, "Classification performance using RF-DNA fingerprinting of ultra-wideband noise waveforms," IET Electronic Letters, vol. 51, no. 10, pp. 787-789, May 2015.

[7] M.W. Lukacs, P.J. Collins, and M.A. Temple, "Device classification using stimulated "RF-DNA" fingerprinting," in Proceedings of 2015 International Symposium on Antennas and Propagation and North American Radio Science Meeting, July 2015, p. to be published.

[8] J.A. Priestly, "AFIT NoNet Enhancements: Software Model Development and Optimization of Signal Processing Architecture," M.S. thesis, Air Force Institute of Technology, Dayton, OH, Mar 2011.

[9] E. Walton, I. Theron, and S. Gunawan, "UWB noise radar using a variable delay line," in 19th Annual Meeting and Symposium of the Antenna Measurement Techniques Association, Boston, MA, Nov 1997.

[10] E. Walton, V. Fillimon, and S. Gunawan, "ISAR imaging using UWB noise radar," in 18th Annual Meeting and Symposium of the Antenna Measurement Techniques Association, Seattle, WA, Sep 1996.

[11] E. Walton, "Digital noise radar prototype development," in Proceed ings of the Antenna Measurement Techniques Association 30th Annual Symposium, Boston, MA, Nov. 16-21 2008.

[12] E. Walton and S. Gunawan, "Development and applications of a 16 channel UHF/L-Band noise radar," in Twentieth Annual Meeting and Symposium of the Antenna Measurement Techniques Association, Montreal, Canada, Oct. 26-30 1998.

[13] A. Schmitt, P.J. Collins, A.J. Jr. Terzuoli, and S. Rogers, "Noise radar correlation patterns of human and non-human objects at various look angles," in Proceedings of the Antenna Measurement Techniques Association 31st Annual Symposium, Salt Lake City, UT, Nov. 1-6 2009.

[14] J. Hardin and P.J. Collins, "An exploration of a multi-function waveform for simultaneous RF communications and ranging," in Proceedings of the Antenna Measurement Techniques Association 35th Annual Symposium, Columbus, OH, Oct. 6-11 2013.

[15] R. Wilson and P.J. Collins, "Noise radar as an indoor navigation aide," in Proceedings of the Antenna Measurement Techniques Association 35th Annual Symposium, Columbus, OH, Oct. 6-11 2013.

[16] W.E. Cobb, E.W. Garcia, M.A. Temple, R.O. Baldwin, and Y.C. Kim, "Physical layer identification of embedded devices using RF-DNA fingerprinting," in Military Communications Conference 2010 - MILCOM 2010, Oct 2010, pp. 2168-2173.

[17] R. Duda, P Hart, and D. Stork, Pattern Classification, John Wiley \& Sons, Inc., New York, 2nd. edition, 2001.

[18] W.E. Cobb, E.D. Laspe, R.O. Baldwin, Michael A. Temple, and Y.C Kim, "Intrinsic physical-layer authentication of integrated circuits," Information Forensics and Security, IEEE Transactions on, vol. 7, no. 1, pp. 14-24, Feb 2012

[19] B. Danev, H. Luecken, S. Capkun, and K. El Defrawy, "Attacks on physical-layer identification.," in 3rd ACM Intl Conf on Wireless Network Security (WiSec10), March 2010. 